\title{
Direct Simulation of Pathological Detonations
}

\author{
James B. Anderson and Lyle N. Long \\ Chemistry Department and Aerospace Engineering Department \\ The Pennsylvania State University, University Park, PA 16802 \\ jba@psu.edu and lnl@psu.edu
}

\begin{abstract}
In previous papers we have demonstrated how the direct simulation Monte Carlo method can be used to simulate detonations. Those simulations were limited to exothermic reactions. In this paper exothermic and endothermic reactions are simulated. Under these circumstances, one obtains pathological detonations. That is, these simulations cannot be predicted using the well-known Chapman-Jouguet hypothesis. The details of these simulations can be illustrated using Rankine-Hugoniot plots and by presenting plots of other quantities of the detonation process. In addition, we describe how the detonation velocity can be changed significantly when endothermic and exothermic reactions are both present.
\end{abstract}

\section{INTRODUCTION}

Detonations are reaction-driven shockwaves that travel at supersonic speeds ${ }^{1-8}$. One-dimensional detonations are fairly well understood and the inviscid 1-D conservation laws provide reasonable predictions of temperature jumps across the detonation and the detonation velocities ${ }^{9}$. These equations lead to the Chapman-Jouguet hypothesis. More complicated time-dependent gas dynamic equations with finite-rate chemistry can be used to simulate the process in more detail; however, these equations are nothing more than the 1-D Euler equations, which cannot adequately model the thermal nonequilibrium of the detonation wave. The 1-D Navier-Stokes equations (with reactions) would also be inadequate. The direct simulation Monte Carlo ${ }^{10,11}$ (DSMC) approach is one of the few approaches that can model the details of a detonation wave.

Kirkwood and Wood ${ }^{12}$ used the term "pathological" to describe gaseous detonations in which the classical Chapman-Jouguet hypothesis might be incorrect. Their extension of the earlier analysis by von Neumann ${ }^{8}$ of the case of a single reaction suggested that "the possibility of pathological behavior may arise, for instance, in gaseous detonations not too strongly exothermic or in detonations in which an exothermic reaction is succeeded by an endothermic one." Dionne et al. ${ }^{13}$ describe pathological detonation experiments using $\mathrm{H}_{2}-\mathrm{Cl}_{2}$. In this case the endothermic reactions are due to dissociating $\mathrm{Cl}_{2}$. Sharpe et al. ${ }^{14,15}$ describe stability analyses of pathological detonations. We report here on DSMC calculations for 1-D detonations which exhibit the behavior suggested. With DSMC and the computers of today, it is possible to explore the details of the entire process, not just the beginning and ending states of the Chapman-Jouguet hypothesis.

\section{COMPUTATIONAL METHOD}

Our calculations are very similar to those we reported earlier ${ }^{9,16}$ for detonations driven by single reactions. In earlier work ${ }^{17-20}$ the use of the DSMC method for reactions is discussed. In the present study we considered several types of reactions including the sequential reactions $\mathrm{A} \mathrm{-->} \mathrm{B} \mathrm{-->} \mathrm{C} \mathrm{in} \mathrm{which} \mathrm{the} \mathrm{first} \mathrm{reaction} \mathrm{occurs} \mathrm{explicitly} \mathrm{as} \mathrm{A}$ $+\mathrm{M}-->\mathrm{B}+\mathrm{M}$ and the second occurs as $\mathrm{B}+\mathrm{M} \mathrm{-->} \mathrm{C}+\mathrm{M}$. The first reaction is exothermic by $8 \mathrm{kcal} / \mathrm{mol}$ and the second is endothermic by 4 or $8 \mathrm{kcal} / \mathrm{mol}$.

The simulations use the DSMC method in one-dimension. We use 1800 cells and roughly 500 particles per cell. While we have run unsteady simulations in the past, the results presented here are from steady-state simulations. Through a simple trial-and-error process the incoming flow velocity is adjusted so that the shock location remains 
fixed. The results do not change if we run an unsteady code and perform ensemble averaging. A no-time-counter collision routine is used also. The cell size is chosen to be roughly 0.5 mean free paths at the incoming flow conditions. In order to simplify the analyses and avoid extraneous effects we treat the molecules as hard spheres of fixed diameter without internal energies. Rotational and vibrational energy models could be included fairly easily in the future. The calculations were carried out in steady-state with an adjustable velocity at the upstream entrance and a vacuum condition at the downstream exit. The feed gas was pure $\mathrm{A}$ at $300 \mathrm{~K}$ at cell number 1800 with flow toward cell number 1 . The molecular masses were specified equal at $20 \mathrm{gm} / \mathrm{mol}$. Reactions were allowed to occur for collision energies exceeding $10 \mathrm{kcal} / \mathrm{mol}$ with steric factors of 1.0 for the first reaction and 0.0 to 1.0 for the second reaction.

The code used here is relatively compact (about 800 lines of Fortran) and was run on a Beowulf (Linux) PC cluster, however the simulations were all run as serial jobs. Each case took about 10 hours to run. We could have run all the simulations as one large job using MPI, and have each processor work on a different case, as described in a previous paper ${ }^{21}$, but we did not do that for this paper.

\section{RESULTS}

The theoretical C-J velocity for the single reaction A --> B is $252,180 \mathrm{~cm} / \mathrm{sec}$ and the observed detonation velocity is essentially the same (within the uncertainty) at $250,000 \mathrm{~cm} / \mathrm{sec}$. Figure 1 shows the computed detonation velocities for several different cases. The solid line shows the results from the sequential reaction A --> $\mathrm{B}$--> C where the first reaction has $8 \mathrm{Kcal} / \mathrm{mol}$ energy release and the second reaction consumes $4 \mathrm{Kcal} / \mathrm{mol}$. The figure shows how the detonation velocity changes with steric factor. With a steric factor of 0.0 , the endothermic reaction does not occur. For a steric factor of 0.1 or higher the second reaction is essentially complete upstream of the exit. For a low steric factor of the endothermic reaction, the reactions are separate, but for large values the reactions are nearly simultaneous. The C-J velocity for a reaction with $4 \mathrm{kcal} / \mathrm{mol}$ energy release is $183,800 \mathrm{~cm} / \mathrm{sec}$ and the observed detonation velocity (depending on the steric factor) varies from 250,000 to $184,000 \mathrm{~cm} / \mathrm{sec}$. The dashed line in Figure 1 is for a simulation with an endothermic reaction of $8 \mathrm{kcal} / \mathrm{mol}$ heat release. In this case the effects are even more pronounced.

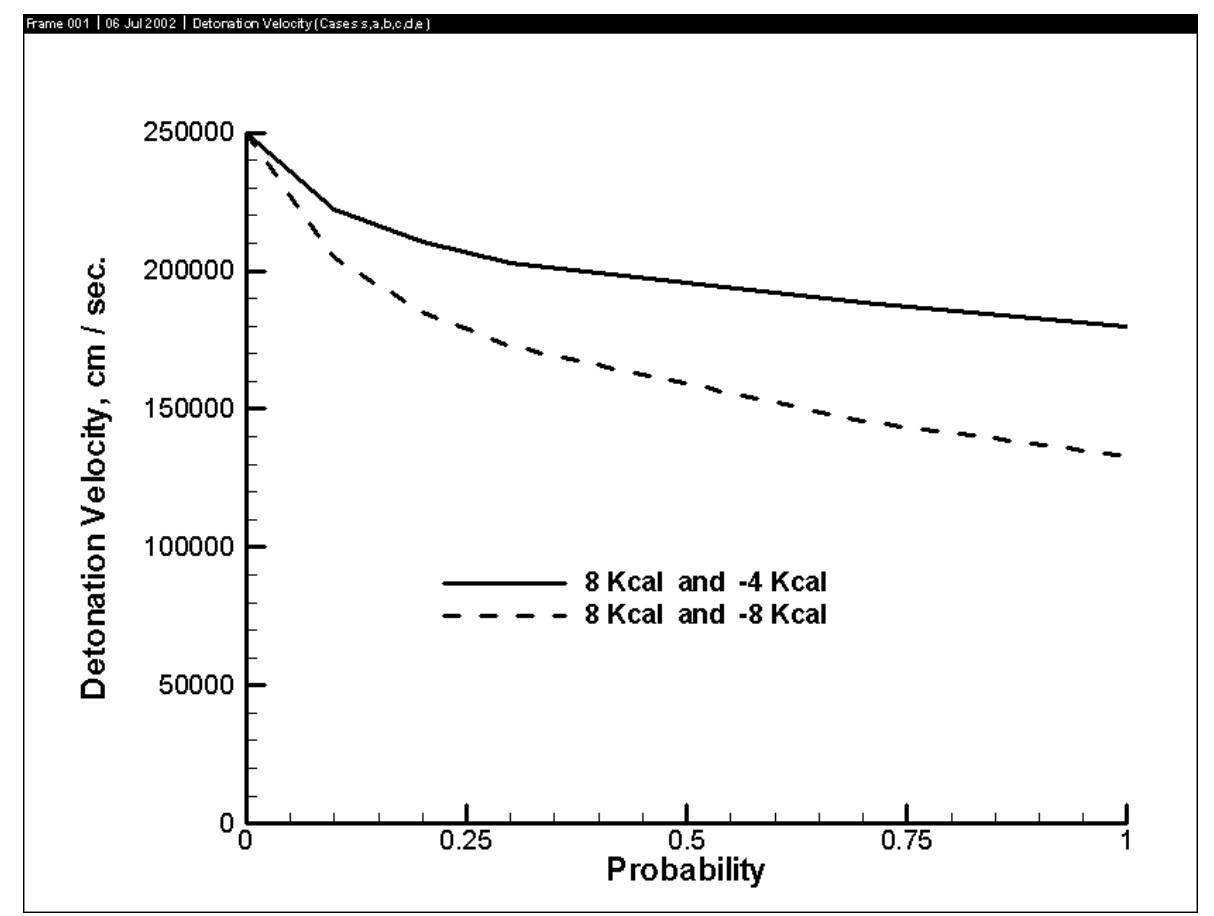

FIGURE 1. Detonation velocity vs. steric factor (probability) for two different endothermic reactions . 
The Rankine-Hugoniot plots for the above cases are shown in Figures 2 and 3. Figure 2 shows graphs of six different values of the steric factor for a case with an exothermic reaction of $8 \mathrm{kcal} / \mathrm{mol}$ and an endothermic reaction of $-4 \mathrm{kcal} / \mathrm{mol}$. All of these plots have the DSMC data shown along with 3 lines of constant heat release. The lower line is the case with no heat release. The middle line is for $4 \mathrm{kcal} / \mathrm{mol}$, and the upper line is for $8 \mathrm{kcal} / \mathrm{mol}$. The detonation process starts on the lower curve at $\mathrm{P} 2 / \mathrm{P} 1=1.0$ and $\mathrm{n} 1 / \mathrm{n} 2=1.0$. We also know ${ }^{6}$, from the Chapman-Jouguet theory, that the final value would be at a point found by drawing a line from the starting point to a point where a tangent curve would intersect the appropriate upper line. These two data points (i.e. the jump conditions of the shock) can be obtained from the Chapman-Jouguet theory. Figure 2a is for a steric factor of 0.0, so this is the baseline case with no endothermic reactions, and the ending point does occur at the point where the tangent line meets the $8 \mathrm{kcal} / \mathrm{mol}$ curve. Figure $2 \mathrm{f}$ is for a steric factor of 1.0 so the endothermic reaction is complete. This result is also consistent with the C-J theory, since it ends at the tangent point on the $4 \mathrm{kcal} / \mathrm{mol}$ line. Figures $2 \mathrm{~b}$ through $2 \mathrm{e}$ are for steric factors of 0.1 to 0.7 . Figure 3 presents the same type of data but for an endothermic reaction of $8 \mathrm{kcal} / \mathrm{mol}$. In this case, however, the exothermic and the endothermic reactions are 8 and $8 \mathrm{kcal} / \mathrm{mol}$ heat release, respectively. Figure 3a is the same as Figure 2a, since they both have a steric factor of 0.0. In Figure $3 \mathrm{f}$ the simulation path leaves the $0 \mathrm{kcal} / \mathrm{mol}$ line and almost returns to it. The detonation velocities associated with the simulations shown in Figure $3 \mathrm{a}$ through $3 \mathrm{f}$ are shown in the dashed line in Figure 1.

The species density and temperature profiles through the shock are shown in Figures 4, 5, and 6. The number densities through the shockwave are labeled $\mathrm{n} 1, \mathrm{n} 2, \mathrm{n} 3$, and $\mathrm{n}$ which denote the concentrations of the species $\mathrm{A}, \mathrm{B}$, $\mathrm{C}$, and the total number density; respectively. In Figure 4, for a steric factor of 0.0, species $\mathrm{C}$ is never produced, and there is a steady increase in species B through the detonation. Figure 5 shows the results for 8 kcal exothermic combined with a $-4 \mathrm{kcal} / \mathrm{mol}$ endothermic reaction and a steric factor of 1.0. In this case there is a steady reduction in species A through the detonation, and a steady rise in species C. Species B only occurs inside the detonation. Figure 6 shows the results when the endothermic and exothermic reactions both have heat release magnitudes of 8 $\mathrm{kcal} / \mathrm{mol}$. In this case the temperature remains fairly low, and therefore species B is not completely consumed.

The temperature curves are labeled Tx and Ty. Tx is the temperature in the flow direction, and Ty is the temperature in a direction normal to the flow direction. As may be seen in Figures 4, 5, and 6, there are significant thermal nonequilibrium effects in the detonations. The results in Figure 4 are for a steric factor of 0.0 (no endothermic reactions), and show peak temperatures near $4000 \mathrm{~K}$. As the endothermic reaction strength is increased, the temperature falls, as shown in Figures 5 and 6, but they do not reach thermal equilibrium.

Figure 7 is shown for completeness. This shows both a Rankine-Hugoniot plot and a temperature plot for an exothermic reaction with a heat release of $4 \mathrm{kcal} / \mathrm{mol}$. This is a traditional detonation wave. The ending point for the solution lies at the tangent point on the $4 \mathrm{kcal}$ curve (of a line drawn from the starting point), as expected.

Figure 8 shows a very interesting case, which illustrates the effect of solely an endothermic reaction. This case starts at an elevated temperature of $2048 \mathrm{~K}$ with a flow speed of $184,000 \mathrm{~cm} / \mathrm{sec}$., with only species B present. The reaction is a $\mathrm{B}+\mathrm{M} \rightarrow \mathrm{C}+\mathrm{M}$ and has a heat release of $-4 \mathrm{kcal} / \mathrm{mol}$. In this case the reaction was delayed until the feed gas reached cell 1700; such that the simulation was, in effect, that of an endothermic reaction following the simple exothermic reaction of the above case. The entering density, temperature, and velocity were specified as those of the freshly formed species B in that case. The gas rapidly cools as the reactions occur. This case illustrates what happens in a pathological detonation: there is an endothermic reaction front that follows immediately behind the detonation wave.

Figure 9 shows the results from a case that has an exothermic $(8 \mathrm{kcal} / \mathrm{mol})$ and an endothermic $(-4 \mathrm{kcal} / \mathrm{mol})$ reaction, however a time delay is used in the endothermic reaction. So the flow involves a traditional detonation, which terminates on the $8 \mathrm{kcal}$ curve. The process then follows the constant energy curve ( $8 \mathrm{kcal})$, but it deviates slightly from the curve due to thermal nonequilibrium and downstream effects. The endothermic reactions begin at the point where there is a slight knee in the curve, and the endothermic reaction takes the process back to the 4 kcal curve. The temperature plot shows a traditional detonation followed by an endothermic reaction front. 

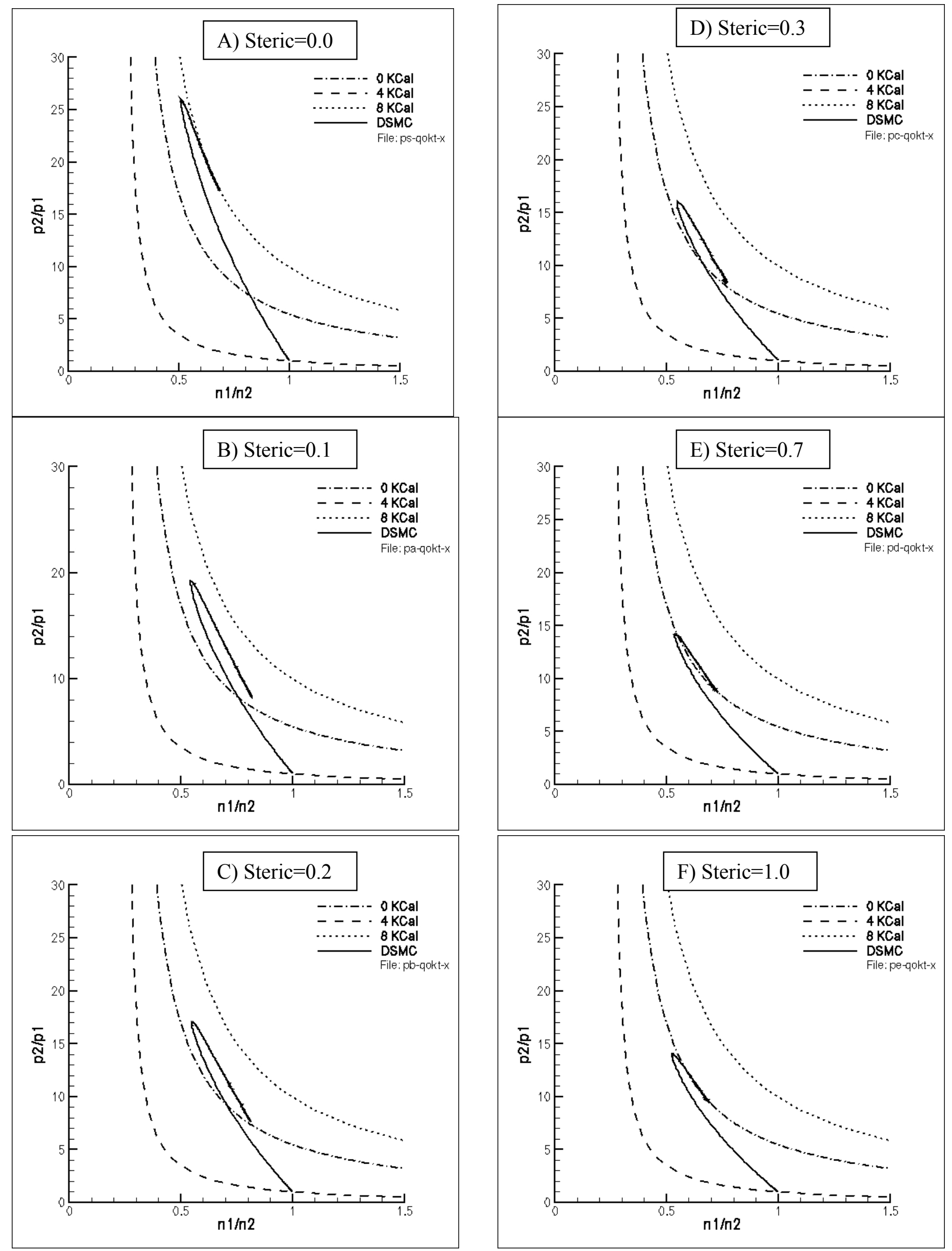

Figure 2. Rankine-Hugoniot plots for $8 \mathrm{kcal} / \mathrm{mol}$ exothermic and $-4 \mathrm{kcal} / \mathrm{mol}$ endothermic reactions for various steric factors: (A) 0.0, (B) 0.1, (C) 0.2, (D) 0.3, (E) 0.7, (F) 1.0. 

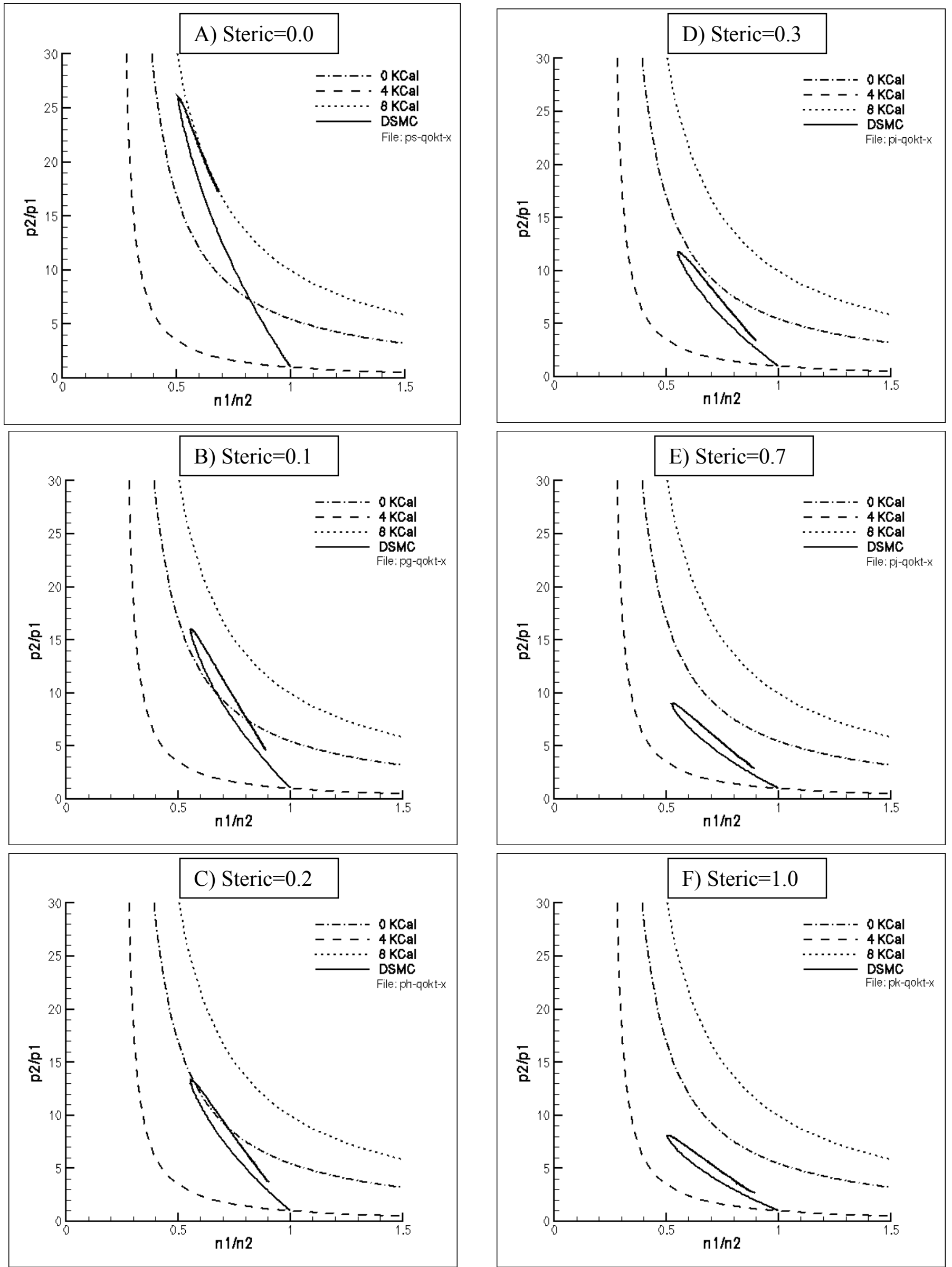

Figure 3. Rankine-Hugoniot plots for $8 \mathrm{kcal} / \mathrm{mol}$ exothermic and $-8 \mathrm{kcal} / \mathrm{mol}$ endothermic reactions for various steric factors: (A) 0.0, (B) 0.1, (C) 0.2, (D) 0.3, (E) 0.7, (F) 1.0. 

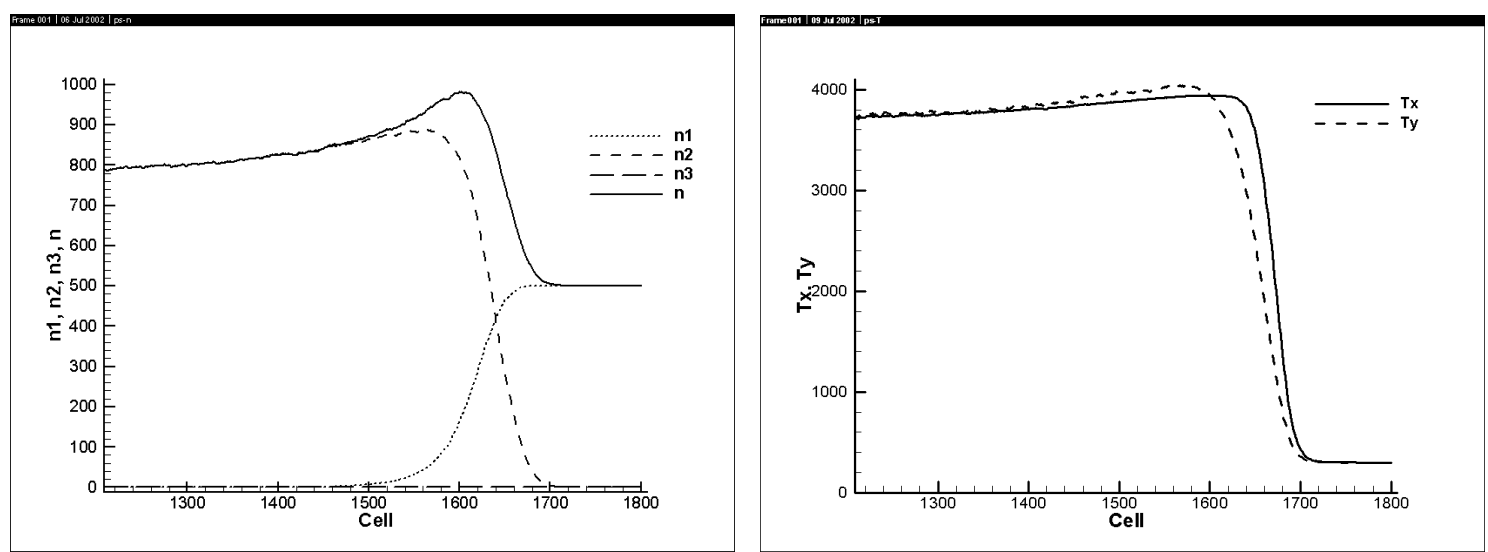

Figure 4. Species concentration and temperature results for steric factor $=0.0(8 \mathrm{kcal} / \mathrm{mol}$ exothermic $)$
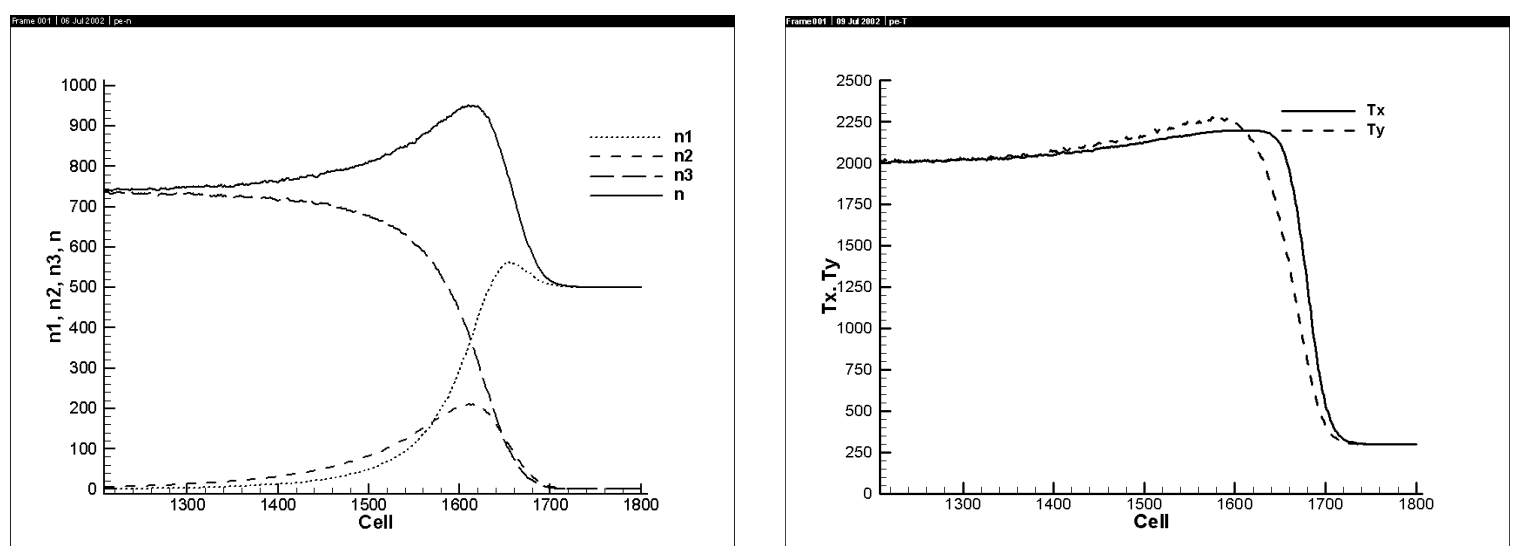

Figure 5. Species concentration and temperature results for steric factor $=1.0 \quad(8 \mathrm{kcal} / \mathrm{mol}$ exothermic and $-4 \mathrm{kcal} / \mathrm{mol}$ endothermic reactions)
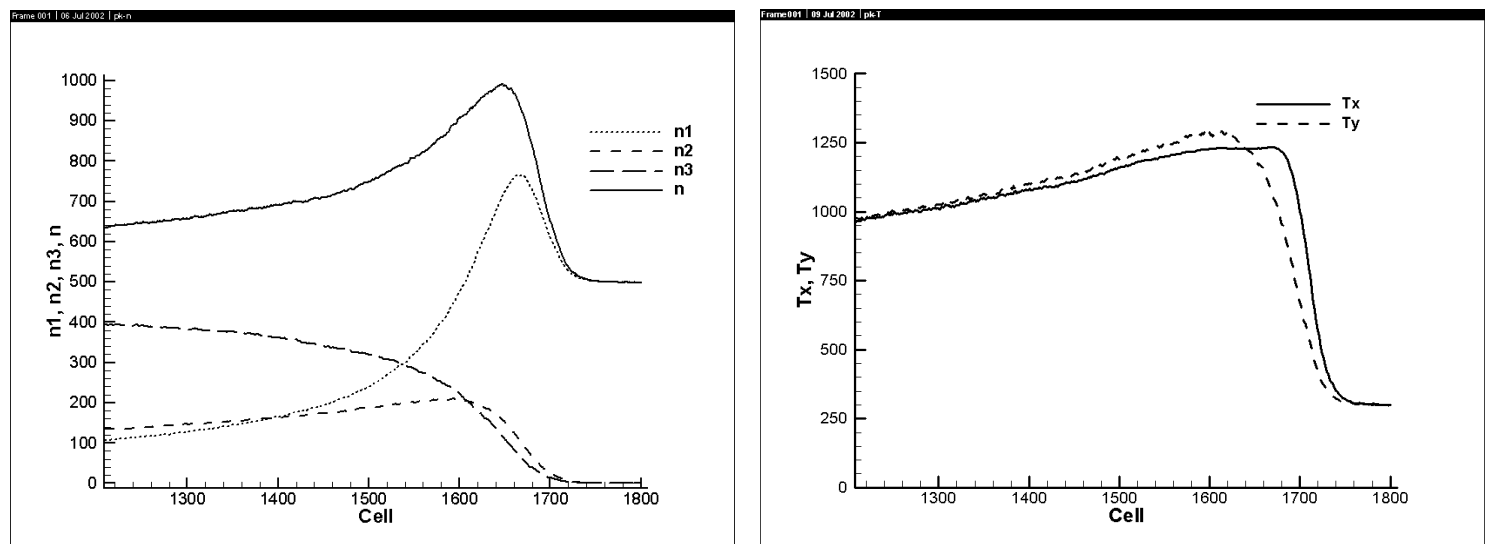

Figure 6. Species concentration and temperature results for steric factor $=1.0 \quad(8 \mathrm{kcal} / \mathrm{mol}$ exothermic and $-8 \mathrm{kcal} / \mathrm{mol}$ endothermic reactions) 

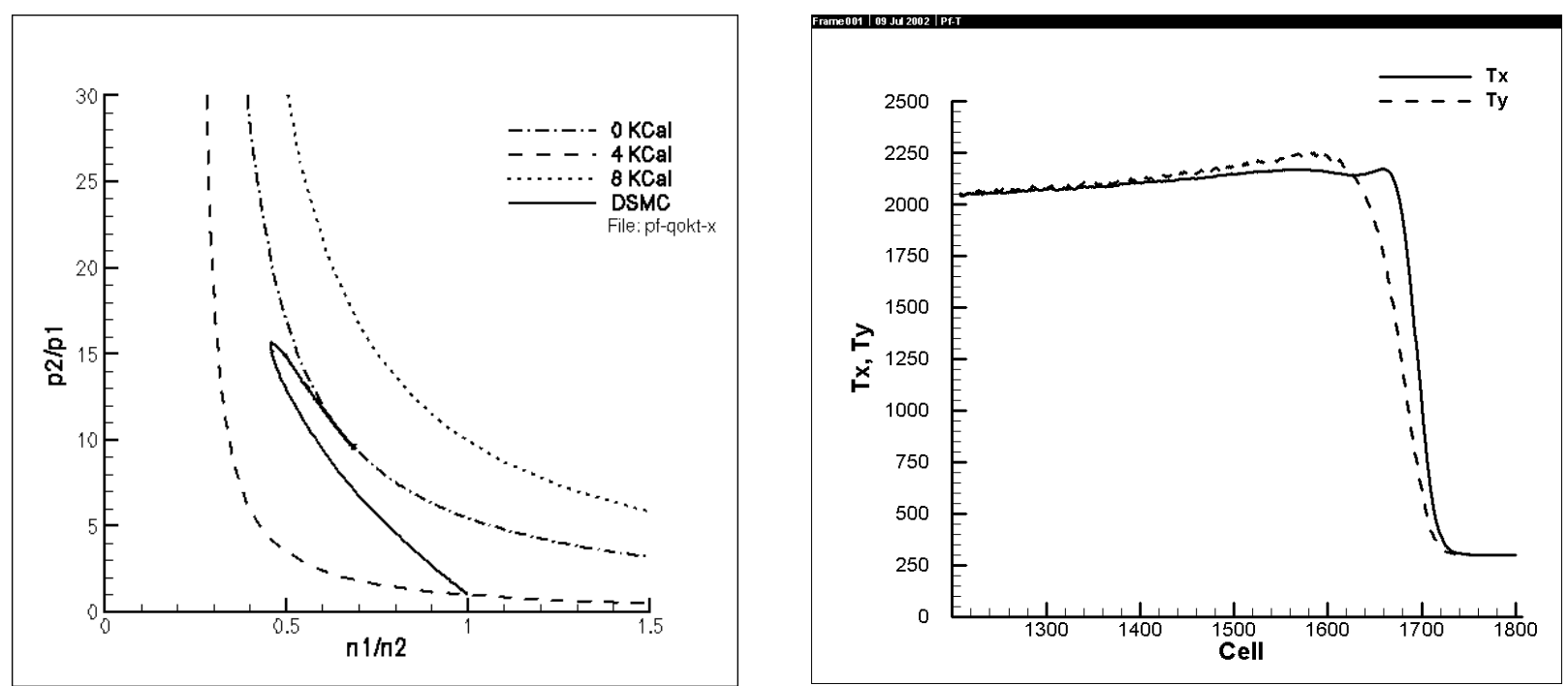

Figure 7. Rankine-Hugoniot plot and temperature results for steric factor $=0.0 \quad(4 \mathrm{kcal} / \mathrm{mol}$ exothermic $)$
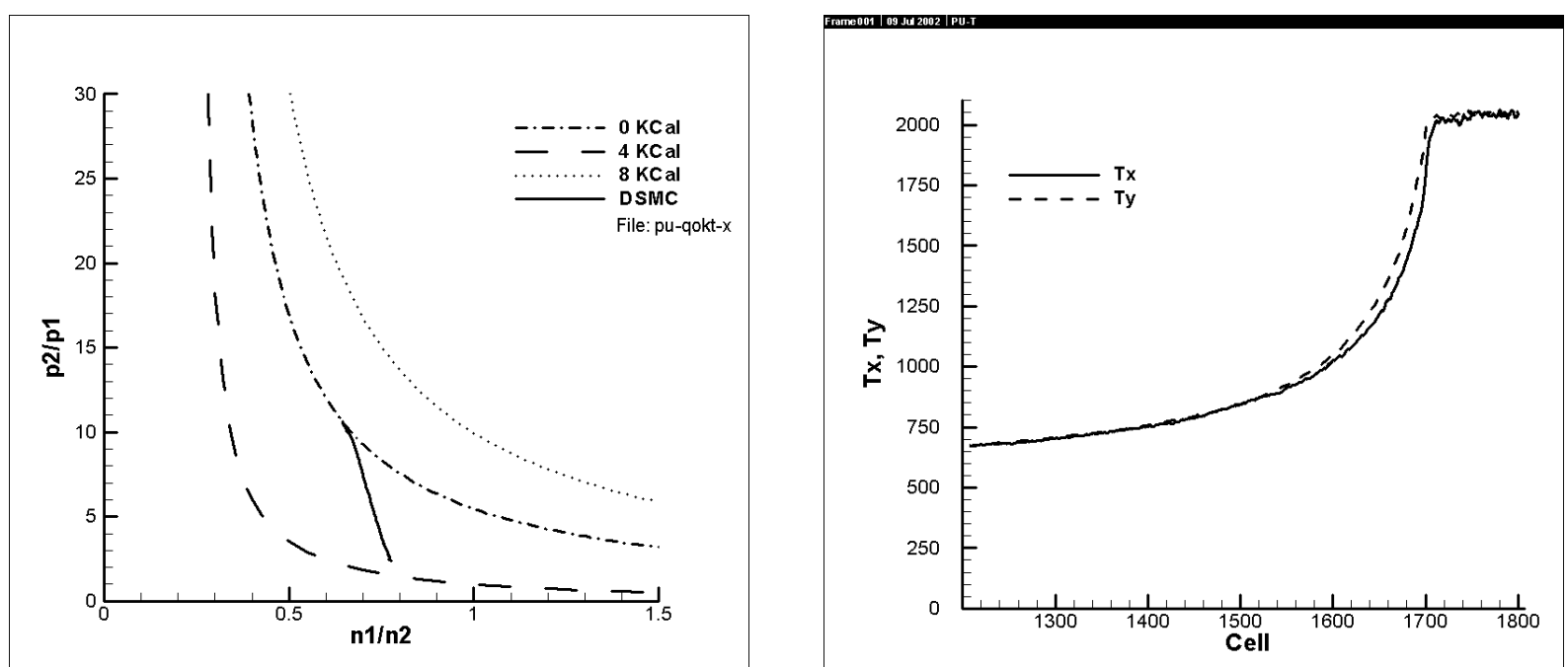

Figure 8. Rankine-Hugoniot plot and temperature results for steric factor $=0.0 \quad(-4 \mathrm{kcal} / \mathrm{mol}$ exothermic $)$
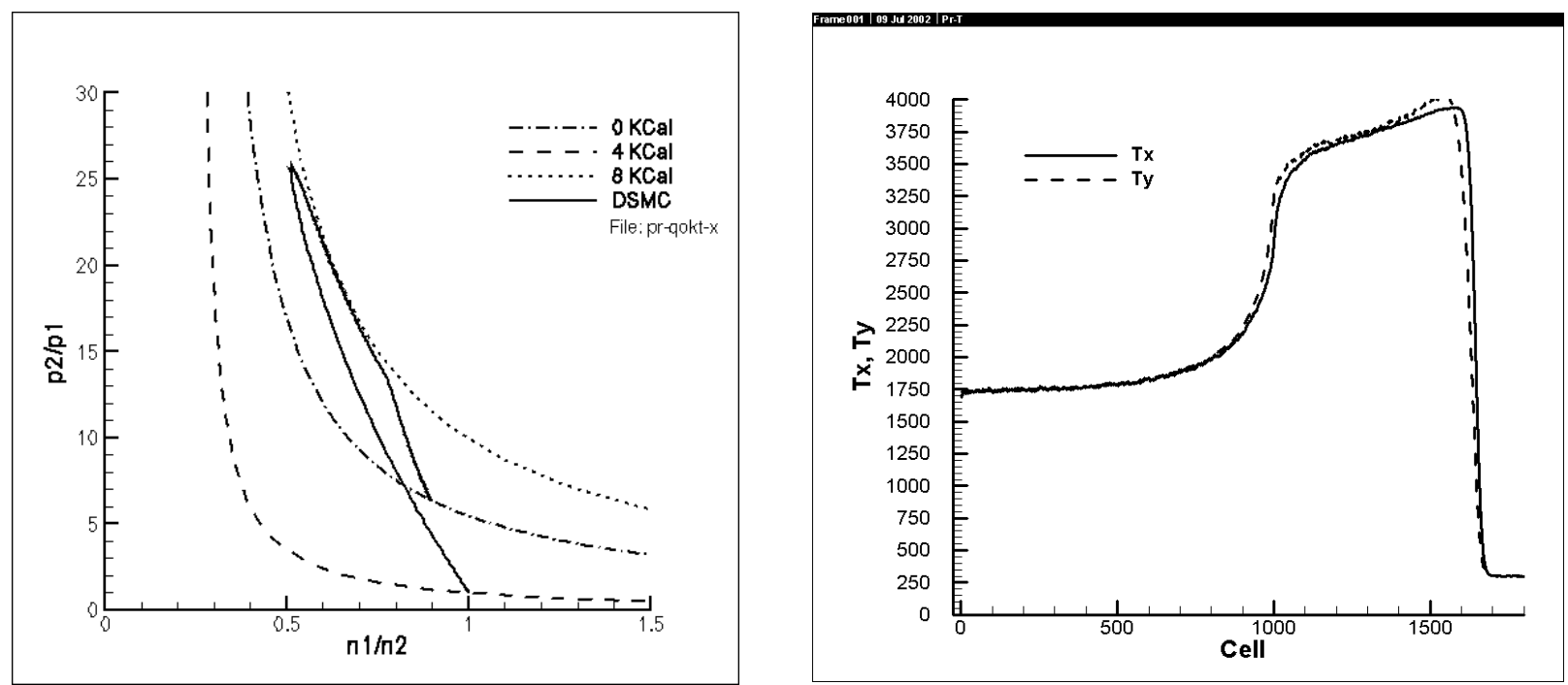

Figure 9. Species concentration and temperature results for steric factor $=1.0(8 \mathrm{kcal} / \mathrm{mol}$ exothermic and 4 $\mathrm{kcal} / \mathrm{mol}$ endothermic, with a spatial delay) 


\section{CONCLUSIONS}

This paper presented results for pathological detonations which have exothermic and endothermic reactions.

These demonstrate that there are coditions under which pathological detonations can occur. In the extreme cases of steric factors of 0.0 and 1.0 the results agree well with the Chapman-Joguet theory. We note the remarkable success in using the DSMC method for pathological detonations, represent classic and extreme examples of the coupling of gas dynamics and chemical reactions. In addition, the effect of the endothermic reactions were isolated and examined.

\section{ACKNOWLEDGMENTS}

We would like to acknowledge support from National Science Foundation Grants: CHE-9734808, CTS9724639, and DGE-9987589. We would also like to thank David A. Long for his help with the postprocessing and graphics.

\section{REFERENCES}

1. D. L. Chapman, Phil. Mag. (London) 47, 90 (1899).

2. E. Jouguet, J. Mathématique 347 (1905); 6 (1906).

3. W. Döring, Ann. Physik. 43, 421 (1943).

4. Y. B. Zeldovich, J. Exptl. Theoret. Phys. (U.S.S.R.) 10, 542 (1940); translated in Natl. Advisory Comm. Aeronaut. Tech. Memo. 1261 (1950).

5. B. Lewis and G. von Elbe, Combustion, Flames and Explosions of Gases, 2nd Edition, Academic Press, New York, pp 511-554 (1961).

6. J. O. Hirschfelder, C. F. Curtiss, and R. B. Bird, Molecular Theory of Gases and Liquids, 2nd Printing, Wiley, New York, pp 797-814 (1964).

7. W. Fickett, Introduction to Detonation Theory, Univ. Calif. Press, Berkeley, pp. 125 (1985).

8. J. von Neumann, O.S.R.D. Report No. 549 (1942), in J. von Neumann, Collected Works, A. H. Taub, Editor, Macmillan Co., New York (1963).

9. L. N. Long and J. B. Anderson, 22nd RGD, Sydney 2000 (2001).

10. G. A. Bird, Phys. Fluids 6, 1518 (1963).

11. G. A. Bird, Molecular Gas Dynamics and the Direct Simulation of Gas Flows (Clarendon, Oxford, 1994).

12. J. G. Kirkwood and W. W. Wood, J. Chem. Phys. 22, 1915 (1954).

13. J. P. Dionne, R. Duquette, A. Yoshinaka, and J.H.S. Lee, Combustion Science and Technology, 158, 5 (2000).

14. G. J. Sharpe, Jnl. Fluid Mechanics, 401, 311 (2000).

15. G. J. Sharpe and S.A.E.G. Falle, Jnl. Fluid Mechanics, 414, 339 (2000).

16. J. B. Anderson and L. N. Long, to appear Jnl. Chem. Phys., 2002.

17. S. D. Piersall and J. B. Anderson, J. Chem. Phys. 95, 971 (1991).

18. S. D. Piersall and J. B. Anderson, Chem. Phys. Lett. 189, 95 (1992).

19. S. M. Dunn and J. B. Anderson, J. Chem. Phys. 99, 6607 (1993).

20. S. M. Dunn and J. B. Anderson, J. Chem. Phys. 102, 2812 (1995).

21. L. N. Long and K. A. Brentner, AIAA Paper 2000-0346, Aerospace Sci. Mtg., (2000). 